\title{
Promovieren in Geschichte und die cotutelle in Frankreich
}

Falk Bretschneider et Christophe Duhamelle

\section{OpenEdition}

\section{Journals}

Édition électronique

URL : http://journals.openedition.org/ifha/7868

DOI : $10.4000 /$ ifha.7868

ISSN : 2198-8943

\section{Éditeur}

IFRA - Institut franco-allemand (sciences historiques et sociales)

Référence électronique

Falk Bretschneider und Christophe Duhamelle, «Promovieren in Geschichte und die cotutelle in Frankreich », Revue de l'IFHA [Online], HS | 2014, Online erschienen am: 01 September 2014 aufgerufen am 02 Mai 2019. URL : http://journals.openedition.org/ifha/7868 ; DOI : 10.4000/ifha.7868

Ce document a été généré automatiquement le 2 mai 2019.

(CIFHA 


\title{
Promovieren in Geschichte und die cotutelle in Frankreich
}

\author{
Falk Bretschneider et Christophe Duhamelle
}

1 Als Historikerin oder Historiker in Frankreich zu promovieren, ist eine hervorragende Idee. Die französische Geschichtswissenschaft gehört (oder gehörte zumindest) zu den bedeutendsten der Welt, seit der Mitte des 20. Jahrhunderts hat sie mit der AnnalesSchule vor allem die Sozial- und Kulturgeschichtsschreibung nachhaltig geprägt ${ }^{1}$. Mit der deutschen Historiographie steht sie seit ihren Anfängen in einem zwar nicht immer intensiven, aber doch kontinuierlichen Austausch. Daneben existiert heute ein einzigartiges Netz an Institutionen der deutsch-französischen Forschungsförderung, das auch und gerade jungen Historikerinnen und Historikern zugutekommt und wissenschaftliches Arbeiten in und zwischen beiden Ländern fast zu einem Kinderspiel macht. Allerdings hat ein Dasein als jeune chercheur in Frankreich - wie überall - auch ein paar Tücken, die man kennen sollte. Der Stellenwert und der soziale Status einer Doktorarbeit gleichen hier nicht den deutschen Gepflogenheiten. Zudem zeigen die Strukturen der Doktorandenausbildung - trotz Bologna-Prozess und fast zwei Jahrzehnten Harmonisierung im europäischen Hochschul- und Forschungsraum - eine Reihe von Eigenheiten, die Uneingeweihten merkwürdig oder zumindest gewöhnungsbedürftig vorkommen können. Die deutsch-französische Doppelbetreuung ( cotutelle de thèse) schließlich ist nicht nur eine attraktive Form des Forschens in einem binationalen Kontext, sondern sie stellt Nachwuchswissenschaftlerinnen und wissenschaftler auch vor das eine oder andere "Kompatibilitäts-Problem«. Aber keine Sorge: Alle diese Herausforderungen sind mit etwas Vorbereitung und Umsicht zu meistern - und dieser Artikel möchte einige Hinweise und Tipps geben, die dabei helfen.

\section{Der soziale Status des Doktorgrades in Frankreich}

2 Mit welchem Prestige der Doktortitel in Deutschland verbunden ist, haben gerade die Plagiatsskandale der letzten Jahre noch einmal deutlich vor Augen geführt. Wer hier die Mühen einer Dissertation auf sich nimmt, strebt nicht unbedingt eine akademische 
Karriere an, sondern hofft mitunter nur auf die soziale Anerkennung und die ökonomischen Vorteile (z. B. ein höheres Einstiegsgehalt), die mit einer Promotion verbunden sind. In Frankreich ist das nicht so - worauf schon die Begriffe hinweisen: Hier benutzt man nicht das Wort promotion (dessen Verwendung auf die Bedeutung »Beförderung« beschränkt ist bzw. den Jahrgang einer grande école bezeichnet, also ein Exzellenzmerkmal, das in Deutschland ohne Äquivalent ist und mit einer Doktorarbeit nichts zu tun hat), sondern spricht vom doctorat ${ }^{2}$. Auch obskure Beratungsfirmen, die gegen Entgelt beim mehr oder weniger legalen Erwerb des Titels behilflich sind, gibt es in Frankreich nicht. Der Doktorgrad ist vielmehr in erster Linie ein akademischer Abschluss, der nötig ist, um eine Berufslaufbahn an Hochschulen oder Forschungseinrichtungen einzuschlagen. Das heißt nicht, dass Promovierte nicht auch in anderen Bereichen tätig sind, z. B. im Schuldienst oder in Archiven, Museen und den Medien ${ }^{3}$. Der Titel ist aber nicht notwendig, um in diesen Berufsfeldern zu arbeiten. Auch öffnet er keine Türen zu Vorstandsetagen. Und für das Gehalt ist er nur insofern relevant, als er eine bestimmte Qualifikationsstufe bezeichnet, die man in Frankreich gern mit der Formel bac +8 (= Abitur plus acht Jahre Studium/Universität) umschreibt.

3 Damit verbunden ist, dass kaum jemand in Frankreich den Titel »trägt«. Wer es dennoch tut, hat gute Chancen, mit einem Arzt verwechselt zu werden, denn Mediziner sind die einzige Berufsgruppe, die ihn - obwohl mitunter nicht einmal promoviert - auch im öffentlichen Raum (also auf Türschildern, Briefpapier oder Visitenkarten) offensiv zur Schau stellt. Ansonsten wird er nur zurückhaltend verwendet, selbst im akademischen Bereich. Hingewiesen wird auf ihn natürlich im Lebenslauf und in Bewerbungsunterlagen (was noch einmal auf seine Funktion als Abschluss hinweist), als Anrede aber ist auch an den Universitäten bestenfalls der Professorentitel üblich. Die in Deutschland weit verbreitete Verwendung akademischer Grade im Alltag würde in Frankreich hingegen als lächerlich empfunden. Hier lässt niemand den Titel in den Ausweis eintragen, erschallt in keiner Hotellobby beim Einchecken des Gastes ein freundliches »Bonjour, Madame le docteur« und erhofft sich kein Wohnungssuchender einen Vorteil gegenüber Mitbewerbern, weil er sich als Promovierter vorgestellt hat. Kurz: Die hohe Wertschätzung, die der Titel in Deutschland in nahezu allen sozialen Milieus genießt, billigt man ihm in Frankreich nicht zu. Außerhalb von Wissenschaft und Forschung kann man mit einem Doktorgrad hier kaum einen Blumentopf gewinnen. Weshalb auch keinem französischen Politiker in den Sinn käme, auf Wahlplakaten als »Monsieur le docteur Dupont « um Stimmen zu werben.

Diese sparsame Verwendung des Doktortitels im gesellschaftlichen Leben ist nicht unbedingt ein Hinweis auf eine vermeintlich egalitärere Kultur in Frankreich, sondern darauf, dass die sozialen Distinktionsregeln und die Mechanismen der Eliteproduktion hier andere sind. Die gesellschaftliche Oberschicht in Wirtschaft, Politik und Verwaltung rekrutiert sich vor allem aus den Absolventen der grandes écoles (die wichtigsten unter ihnen sind: die École Nationale d'Administration, kurz: ENA, die École Polytechnique, kurz: X, die Militärhochschule Saint-Cyr, die Ingenieurhochschule École Centrale, die Wirtschaftshochschule École des hautes études commerciales, kurz: HEC; im Bereich der Human- und Sozialwissenschaften wichtig sind die drei Écoles Normales Supérieures, kurz: ENS oder »Normale Sup«, in Paris, Cachan und Lyon). Die Abschlüsse dieser Einrichtungen kann nur erwerben, wer einen harten Selektionsprozess durchlaufen hat, den zu bestehen neben intellektuellen Fähigkeiten auch das notwendige soziale und symbolische Kapital erfordert. Die soziale Rolle, die in Deutschland dem Doktortitel 
zukommt, wird in Frankreich also zumindest teilweise von der Bezeichnung »ancien élève de ..." übernommen. Die Promotion hingegen stellt - im Vergleich zum deutschen Nachbarn - ein Minderheitenphänomen dar: Während in Deutschland jährlich etwa 25.000 Dissertationen verteidigt werden, sind es in Frankreich aktuell etwa 11.000 (ihre Zahl ist allerdings seit einiger Zeit im Steigen begriffen), wovon etwa ein Viertel auf die Geistes- und Sozialwissenschaften entfälltt ${ }^{4}$. Wer promoviert, signalisiert hier also nicht unbedingt, dass er zur gesellschaftlichen Elite gehören möchte. Gleichzeitig wird, wer zu eben dieser Elite gezählt werden will, nur selten promovieren.

Auf dem Weg zur Karriere als Forscher oder Hochschullehrerin ist die Anfertigung einer Dissertation jedoch links wie rechts des Rheins eine unerlässliche Etappe. Aber auch hier gibt es Unterschiede. In Deutschland, wo die Promotion lange Zeit der einzige Studienabschluss war, steht sie in einer gewissen Kontinuität $\mathrm{zu}$ den anderen Universitätsdiplomen. In Frankreich hingegen bildet sie einen eigenen Abschnitt, was auch mit dem stark verschulten Studium zu tun hat. In einen engeren Kontakt mit der Forschung geraten Studierende an französischen Universitäten oft erst im Master (wenn dieser ein Forschungsprofil aufweist) oder eben im Rahmen der Dissertation. Verbunden damit ist, dass sich in Frankreich leichter als in Deutschland die Idee durchsetzen konnte, dass Doktoranden keine Studierenden mehr sind, sondern bereits selbst aktiv am Forschungsprozess teilhaben. Die frühere Bezeichnung thésard ist in den letzten Jahren deshalb nahezu verschwunden; heute gelten Promovierende für die meisten ganz selbstverständlich als jeunes chercheurs. In Deutschland hingegen spricht man nach wie vor gern von "Nachwuchswissenschaftlern", wobei dieser Status in der informellen Hierarchie der Wissenschaft bis weit über die Dissertation hinaus anhält. Die Botschaft: Wer in diese Gruppe gehört, muss sich seine wirklichen Meriten erst noch verdienen ${ }^{5}$. Das verweist auf den vielleicht wichtigsten Unterschied: Im deutschen System gilt ein frisch gebackener Doktor nicht viel. Denn trotz Juniorprofessur und anderer alternativer Karrierewege warten bis zum Ziel der Festanstellung auf die meisten noch weitere Hürden der Qualifizierung wie die Habilitation. In Frankreich hingegen ist eine junge Doktorin fast schon eine Kollegin, denn eine feste Stelle an Hochschulen oder Forschungseinrichtungen wie dem CNRS winkt hier (zumindest im Moment noch) bereits nach der Promotion.

Unterschiedlich ist schließlich auch der Qualifizierungscharakter der Promotion. Weder in Frankreich noch in Deutschland reicht die Dissertation allein aus, um im akademischen Bereich zu reüssieren. Im deutschen Fall kommen so weitere akademische Kriterien ins Spiel (etwa die Veröffentlichung der Arbeit in einer angesehenen Reihe, die Einwerbung von ersten Drittmitteln oder von Postdoc-Stipendien renommierter Universitäten usw.), die auch in Frankreich zunehmend eine Rolle spielen. Weit wichtiger für Stellenbesetzungen an französischen Universitäten sind aber nach wie vor traditionelle Elemente, die mit dem Beruf der Forscherin oder des Hochschullehrers bestenfalls indirekt zu tun haben, wie etwa das Bestehen der agrégation (Lehramtsprüfung) ${ }^{6}$ oder das Studium an einer grande école. Bemerkbar macht sich hier die bis heute in Frankreich traditionell sehr starke Verknüpfung von Universitätsstudium und Sekundarschulbereich. Von engeren Verbindungen zwischen Lehre und Forschung lässt sich an den französischen Hochschulen hingegen erst in den letzten Jahrzehnten sprechen (was daran liegt, dass die Universitäten in Frankreich lange Zeit vor allem Stätten der Berufsausbildung und nicht der Wissenschaft waren) ${ }^{7}$. Und noch eine französische Besonderheit, die die hier starken zentralstaatlichen Traditionen 
widerspiegelt, muss erwähnt werden: Um sich für feste Stellen an den Universitäten bewerben zu können (nach der Dissertation als Maître de conférences, nach der Habilitation als Professeur) ist es notwendig, sich von einem besonderen Gremium in Paris, dem nach Disziplinen organisierten Conseil National des Universités (kurz: CNU), evaluieren zu lassen (der Fachausdruck lautet »qualification «) ${ }^{8}$. Seine Mitglieder sind zum Teil ernannte, zum Teil gewählte Hochschullehrerinnen und -lehrer aus dem ganzen Land. Die Tätigkeit des CNU bestimmt nicht nur den Rhythmus des akademischen Lebens in Frankreich (die Dossiers müssen in der Regel im Dezember eingereicht werden, weshalb die meisten Dissertationsverteidigungen in der Form eines Marathonlaufs am Ende des Jahres stattfinden), sondern auch den Wert des Diploms an sich: Ein nicht unbeträchtlicher Teil der Promovierten (in Geschichte etwa die Hälfte derjenigen, die sich um eine » qualification « beworben haben) besitzt zwar den Doktorgrad, hat aber - da das CNU eine positive Evaluierung versagt - keine Möglichkeit, sich auf eine Stelle als Maitre de conférences zu bewerben.

\section{Strukturen der Doktorandenausbildung}

7 Wie es sich für einen Zentralstaat gehört, sind die Strukturen der Doktorandenausbildung in Frankreich national organisiert. Während in Deutschland der Rechtsrahmen der Promotion, die administrativen Regelungen und auch die Noten von Land zu Land, ja von Universität zu Universität und mitunter sogar von Fakultät zu Fakultät variieren können, gibt es hier eine einheitliche gesetzliche Grundlage und die Promotion ist ein vom Staat verliehenes, nationales Diplom. Lange Zeit hatte es zwei aufeinander aufbauende französische Doktorgrade gegeben: den docteur de troisième cycle und den docteur d'État (der in den Geistes- und Sozialwissenschaften zum Titel docteur ès lettre führte). Mit einem 1984 erlassenen Gesetz (Loi Savary, nach dem damaligen Hochschulminister Alain Savary) löste die Habilitation (habilitation à diriger des recherches oder abgekürzt: HDR) die zweite Stufe $\mathrm{ab}$, während die erste nun als doctorat de nouveau régime bezeichnet wurde. Seitdem gilt auch in Frankreich die international übliche Zweiteilung der akademischen Qualifikationen in Promotion und Habilitation. Im Zuge des Bologna-Prozesses wurde mit einer Verordnung vom 7. August 2006 die französische Doktorandenausbildung schließlich erneut reformiert und den internationalen Gepflogenheiten weiter angepasst ${ }^{9}$ . Als Titel wird heute in der Regel nur noch der Grad docteur verliehen, eventuell gefolgt von der Disziplin und einer Spezialisierung (also z. B. docteur en histoire, mention histoire médiévale). Daneben ist das bislang der Promotion vorausgehende diplôme d'études approfondies (DEA) weggefallen bzw. durch den Master 2 recherche (Masterdiplom mit explizitem Forschungsbezug) ersetzt worden.

8 Ebenfalls 2006 eingeführt wurde die Verpflichtung aller Hochschulen, eine strukturierte Doktorandenausbildung anzubieten. Das bedeutete zunächst vor allem die flächendeckende Einrichtung von "Doktorandenschulen" (écoles doctorales), in die sich einzuschreiben alle Promovierenden verpflichtet sind und die nunmehr allein über die Verteilung von Promotionsstipendien (allocations doctorales) entscheiden. Die Struktur und das Ausbildungsangebot jeder dieser Doktorandenschulen werden staatlich geprüft und genehmigt. Ziel ist eine "Ausbildung durch Forschung" ("formation par la recherche«), die junge Wissenschaftlerinnen und Wissenschaftler befähigt, am Ende der Promotionszeit eine eigenständige Forschungsleistung vorzulegen. Jeder Doktorand ist deshalb nicht nur in die zuständige Doktorandenschule integriert, sondern auch Mitglied 
in der Forschungseinheit seines Betreuers bzw. seiner Betreuerin (équipes d'accueil in den Universitäten, unités mixtes de recherche oder UMR zwischen Hochschuleinrichtungen und dem nationalen Forschungszentrum CNRS usw.). Damit soll sichergestellt werden, dass die Promotion nicht nur einen Ausbildungsabschnitt darstellt, sondern zudem eine erste selbstständige Berufserfahrung. Mit den gleichen Absichten verbunden ist auch die politische Zielvorgabe, Doktoranden möglichst nur noch im Rahmen von speziellen Arbeitsverträgen promovieren zu lassen (siehe unten). Von den Doktorandenschulen angeboten wird darüber hinaus ein pädagogisches Programm, das meist aus Forschungsund Methodenseminaren sowie speziellen Ausbildungsmodulen besteht (Schreib- und Sprachkurse, oft auch Wissenschaftsfranzösisch für Ausländer, Einführungen in die neuen Medien, Sommerschulen usw.). Auch werden die Doktoranden angehalten, erste Erfahrungen in der Organisation wissenschaftlicher Veranstaltungen (Ateliers usw.) sowie beim Publizieren zu machen. Über die individuelle Betreuung durch Doktorvater oder -mutter hinaus soll ihnen also eine kollektive Forschungsumgebung angeboten werden, die sie mit den Gepflogenheiten wissenschaftlichen Arbeitens vertraut macht, gleichzeitig aber auch für eine mögliche Tätigkeit auf dem nicht akademischen Arbeitsmarkt vorbereitet.

Natürlich variieren die Angebote der einzelnen Doktorandenschulen erheblich. In den meisten Universitäten sind sie nach Disziplinen gegliedert, so dass die Historiker unter Historikern und die Soziologen unter Soziologen bleiben. Einige Hochschulen - etwa die École des hautes études en sciences sociales (EHESS) ${ }^{10}$ - bieten aber auch interdisziplinäre Doktorandenschulen an, innerhalb derer es möglich ist, sich mit Promovierenden anderer Fächer auszutauschen. Daneben existieren einige besondere Strukturen, die speziell auf eine binationale Doktorandenausbildung hin ausgerichtet sind. Dazu gehören zuerst die Deutsch-Französischen Doktorandenkollegs, die von der Deutsch-Französischen Hochschule (DFH) eingerichtet und von mindestens zwei Partnereinrichtungen in Deutschland und Frankreich getragen werden. Wer in diese Kollegs eingeschrieben ist, kann nicht nur auf finanzielle Unterstützung für einen Forschungsaufenthalt im Partnerland zählen, sondern kommt auch in den Genuss von Ausbildungsangeboten, die den Erwerb von methodisch-reflexiven, interdisziplinären und interkulturellen Kompetenzen in den Mittelpunkt stellen. Außerdem ist jedes Kolleg einer bestimmten wissenschaftlichen Thematik gewidmet. Sie sind also eine hervorragende Möglichkeit, um sich frühzeitig in ein deutsch-französisches Forschungsnetzwerk zu integrieren und die eigene wissenschaftliche Arbeit in einen permanenten interkulturellen Austausch einzubetten. Derzeit werden von der DFH etwa zwei Dutzend solcher Kollegs angeboten, von denen mehrere auch geschichtswissenschaftliche Themen betreffen (z. B. die Kollegs zwischen der EHESS und der Humboldt-Universität Berlin oder zwischen der Universität Paris 1 und der Goethe-Universität Frankfurt a. M.) ${ }^{11}$.

10 Eine weitere Initiative außerhalb der Doktorandenschulen an den Hochschulen ist das deutsch-französische Doktorandenbetreuungsprogramm am Pariser Centre interdisciplinaire d'études et de recherches sur l'Allemagne (CIERA), einem vom DAAD und dem französischen Hochschulministerium geförderten Netzwerk aus elf französischen Universitäten, Hochschulen und Forschungseinrichtungen. Angeboten wird hier ein auf die spezifischen Anforderungen des interkulturellen, deutschfranzösischen Promovierens hin ausgerichtetes Programm aus allgemeinen Methodenseminaren (»Einführung in das Promovieren«, "Vorbereitung auf die Verteidigung" usw.) sowie speziellen Ausbildungsmodulen (Schreibateliers, Arbeiten mit 
elektronischen Ressourcen). Darüber hinaus gibt es fachspezifische Veranstaltungen, aber auch interdisziplinäre Doktorandenkolloquien und Sommerschulen. Schließlich können im Rahmen der Zeitschrift »Trajectoires - Travaux des jeunes chercheurs du CIERA« erste Erfahrungen im Publizieren gemacht werden, und mit den Colloques Junior wird die Organisation einer ersten wissenschaftlichen Tagung gefördert. Im Zusammenspiel mit den übrigen wissenschaftlichen Aktivitäten des Zentrums (z. B. den Programmes de formation-recherche) offeriert das CIERA also eine einzigartige, interdisziplinär und interkulturell ausgerichtete Forschungsumgebung, die auch und gerade für junge Historikerinnen und Historiker interessant ist ${ }^{12}$.

11 Mit Stipendien, der Organisation von Nachwuchsgruppen, Fachsprachkursen und Sommerschulen fördert auch das Deutsche Historische Institut Paris Doktorandinnen und Doktoranden in Geschichte ${ }^{13}$. Die Bibliothek des Instituts stellt darüber hinaus die wichtigste Forschungsbibliothek zur deutsch-französischen Geschichte dar - sie ist auch für Nicht-Institutsmitglieder frei zugänglich. Und auch sein Pendant in Deutschland, das Institut français d'histoire en Allemagne in Frankfurt, organisiert Veranstaltungen, die sich besonders an junge Historikerinnen und Historiker richten, und schreibt regelmäßig Doktorandenstellen aus (z. B. im Rahmen des Verbundprojekts "Saisir l'Europe - Europa (be-)greifen", an dem u. a. auch das CIERA, das DHI Paris und das deutsch-französische Centre Marc Bloch beteiligt sind) ${ }^{14}$.

Besondere Unterstützung bei der Berufseingliederung auf dem nicht akademischen Arbeitsmarkt bietet die bei der DFH angesiedelte deutsche Antenne der in Paris ansässigen ABG-L'intelli'agence (vormals Association Bernard Grégory) an. Hier stehen spezifische Coachingprogramme (z. B. »Interkulturelles Bewerbertraining«) sowie Hilfen beim Aufbau von Netzwerken (»Apéro Doc«) im Mittelpunkt ${ }^{15}$. Schließlich existieren auch einige wenige Initiativen, die von Doktorandinnen und Doktoranden selbst entwickelt wurden - etwa das Seminar "Les aspects concrets de la thèse« an der EHESS. Dort können Promovierende ihre Erfahrungen beim wissenschaftlichen Arbeiten untereinander, aber auch mit Gästen diskutieren. Zur Sprache kommen dabei nicht nur »klassische« Themen wie Schreibblockaden, die Arbeitsorganisation oder das richtige Zitieren, das Seminar will jüngere und erfahrene Wissenschaftlerinnen und Wissenschaftler auch $\mathrm{zu}$ einem gemeinsamen Nachdenken über die Praxis der Wissenschaft anregen. Verbunden mit diesen Aktivitäten ist inzwischen ein Weblog, so dass die Diskussionen auch online geführt werden können ${ }^{16}$. Auch im Rahmen der von jungen Wissenschaftlerinnen und Wissenschaftlern getragenen deutsch-französischen Gruppe GIRAF-IFFD werden vergleichbare Hilfestellungen angeboten, insbesondere für Doktorandinnen und Doktoranden in Doppelbetreuung ${ }^{17}$.

13 Schließlich gibt es auch in Frankreich eine zunehmend breite Palette von Ratgeberliteratur zum Promovieren. In der Regel beziehen sich die dort gegebenen Tipps und Hilfestellungen auf die administrativen Abläufe und auf typische wissenschaftliche Arbeitstechniken (Bibliographieren, Arbeit mit dem Computer, Zitieren, Zeitmanagement, usw. $)^{18}$. Für Nicht-Franzosen hilfreich können solche Ratgeber vor allem deshalb sein, weil hier auch die Besonderheiten des wissenschaftlichen Schreibens im französischen Hochschulsystem erklärt werden. Französische Studierende lernen etwa die berühmt-berüchtigte Einteilung der Argumentation in drei Teile (die längst nicht mehr so streng angewandt wird wie noch vor ein paar Jahren, ihren stilbildenden Einfluss aber auch nicht gänzlich verloren hat) oder die traditionelle Aufstellung eines plan de rédaction von der Pike auf. Wer in einem anderen System mit anderen rhetorischen 
Traditionen groß geworden ist, wird hingegen bei der Anwendung dieser vergleichsweise strengen Regeln womöglich auf einige Schwierigkeiten stoßen. Sich mit ihnen frühzeitig vertraut zu machen, kann deshalb nur empfohlen werden.

\section{Promotionsbedingungen konkret}

Damit sind wir schon bei den konkreten Bedingungen, die das Promovieren in Frankreich bestimmen. Beginnen wir mit den Formalien: Die Einschreibung in das Doktorat ist zwingend mit der Registrierung des gewählten Themas im "Zentralregister der Dissertationen « (Fichier central des thèses, FCT) verbunden, das von der Agence bibliographique de l'Enseignement supérieur (ABES) verwaltet wird ${ }^{19}$. Dafür notwendig sind die Zustimmung des Betreuers, des Leiters der Doktorandenschule und oft auch des Präsidenten der Hochschule. Ebenfalls zur Einschreibung gehört die Unterzeichnung einer Dissertations-Vereinbarung (Charte de thèse), in der die Rechte und Pflichten von Promovend, Betreuer und Doktorandenschule festgelegt sind. Die konkreten Bestimmungen dieses Dokuments können von Hochschule zu Hochschule leicht variieren, beruhen im Prinzip aber auf zentralen Vorgaben ${ }^{20}$. Daneben sind mit der Einschreibung Gebühren verbunden (droits d'inscription), deren Höhe ebenfalls zentral festgelegt wird (2013: $388 €)$, sowie Beiträge zur studentischen Krankenversicherung und zur medizinischen Versorgung in der Hochschule ${ }^{21}$. Schließlich sollte, wer seine Dissertation in deutsch-französischer Doppelbetreuung anfertigen möchte (siehe unten), die entsprechenden administrativen Schritte so frühzeitig wie möglich in die Wege leiten.

Die Dauer einer Dissertation ist in Frankreich verbindlich auf drei Jahre festgeschrieben. Im Laufe dieser Zeit müssen sich Promovenden jedes Jahr neu in ihre Hochschule einschreiben. Da alle Beteiligten wissen, dass gerade in den Geistes- und Sozialwissenschaften drei Jahre für die Fertigstellung der Arbeit weitgehend illusorisch sind, gibt es die Möglichkeit, sich nach Ablauf dieser regulären Promotionszeit ein viertes Mal einzuschreiben, um die Arbeit zu verteidigen (auch die dreijährigen contrats doctoraux können auf Antrag um ein Jahr verlängert werden). Zur Verteidigung (soutenance) zugelassen wird eine Arbeit vom Präsidenten der Hochschule nach einer Beurteilung des Manuskripts durch zwei auswärtige Gutachter, die auf Vorschlag des Betreuers der Arbeit ausgewählt wurden. Auch die Promotionskommission (jury de thèse) wird vom Leiter der Hochschule auf Vorschlag des Betreuers berufen (im Idealfall stimmen sich Betreuer und Promovend in diesen Fragen ab). Mindestens die Hälfte der Mitglieder der Kommission muss von außerhalb kommen (dazu zählen in der Regel auch die beiden auswärtigen Gutachterinnen oder Gutachter). Im Unterschied zu Deutschland können in Frankreich auch Wissenschaftler unterhalb des Professorenrangs (also vor allem Maîtres de conférences an den Universitäten und Chargés de recherches am CNRS) an einer Promotionskommission teilnehmen (ihre Zahl darf allerdings die Hälfte der Mitglieder nicht übersteigen). Die Verteidigung findet öffentlich statt und endet bei Annahme der Dissertation mit einem der drei folgenden Prädikate: honorable, très honorable oder très honorable avec félicitations. Da das höchste Prädikat in Frankreich seit langem weitgehend inflationär vergeben wird, sind für die Verleihung der félicitations seit einiger Zeit eine geheime Abstimmung und eine gesonderte Begründung notwendig. Dennoch erhalten die meisten Kandidaten weiterhin die Bestnote (was auch heißt, dass, wer sie nicht vorweisen kann, kaum noch Chancen auf eine Karriere im akademischen Bereich hat). 
16 Nach der Verteidigung wird vom Vorsitzenden der Kommission ein Bericht (rapport de soutenance) erstellt, der von allen Kommissionsmitgliedern unterzeichnet werden muss. Dieses Dokument ist den deutschen Promotionsgutachten vergleichbar und von allerhöchster Wichtigkeit für alle, die ihre Berufslaufbahn an der Hochschule oder in der Forschung fortsetzen möchten. Es muss allen Bewerbungen beiliegen (inklusive jener um die qualification beim CNU) - denn da die Note der Dissertation nur bedingt über ihre wissenschaftliche Qualität Auskunft gibt, lässt sich nur aus den detaillierten Beurteilungen herausdestillieren, was die Mitglieder der Promotionskommission tatsächlich von der Arbeit gehalten haben. Dabei gilt, dass in Frankreich Kritik in der Regel nur verhalten geäußert wird. Während in Deutschland die Patzer eines Kandidaten schon einmal offen zur Schau gestellt werden, zählen hier eher die verschiedenen rhetorischen Abstufungen der Begeisterung. Negative Bewertungen stehen also gern zwischen den Zeilen oder verstecken sich in neutralen Formulierungen. Nicht unüblich ist es hingegen, detailreich Grammatik- und Orthographiefehler aufzuzählen oder aber, im Gegenteil, die stilistischen Qualitäten der Arbeit in aller Ausführlichkeit zu loben.

17 Daneben muss die Kommission Stellung nehmen, ob das Manuskript in der vorliegenden Form vervielfältigt werden kann oder aber überarbeitet werden muss. Im letzten Fall stehen dafür drei Monate zur Verfügung, bevor es auf Staatskosten vom Atelier national de reproduction auf Mikrofiche verfilmt und den französischen Universitätsbibliotheken zur Verfügung gestellt wird. Seit einiger Zeit können die verfilmten Manuskripte auch frei über das Internet erworben werden ${ }^{22}$. Eine darüber hinausgehende Veröffentlichungspflicht besteht nicht. Gute Dissertationen erscheinen aber auch in Frankreich zunehmend im Druck - in der Regel jedoch erst nach einer gründlichen inhaltlichen und sprachlichen Überarbeitung, die aus dem Manuskript ein Buch macht, das auch Leserinnen und Leser über den engeren Kreis der Spezialisten hinaus anspricht.

Hinsichtlich der materiellen Konditionen für Doktorandinnen und Doktoranden sind vor allem zwei Elemente von Bedeutung: Zum einen wird von politischer Seite seit einigen Jahren erheblicher Druck auf die Hochschulen ausgeübt, Promotionen nur noch im Rahmen von Arbeitsverträgen zuzulassen. Das geht auf eine Direktive der Europäischen Kommission zurück, die in Deutschland bislang nicht umgesetzt wurde. Reagiert wurde damit u. a. auf den Umstand, dass Doktorandenstipendien in Frankreich äußerst rar gesät sind, Promovierende also vor allem auf Nebenjobs oder elterliche Hilfe angewiesen sind, um ihre Dissertation zu finanzieren - so sie denn nicht zu denen zählen, die das absolvieren, was von vielen nach wie vor als "Königsweg" einer Karriere als jeune chercheur angesehen wird: der Erwerb der agrégation und anschließend eine Tätigkeit als verbeamteter Gymnasiallehrer bzw. als Hilfskraft (z. B. als moniteur oder attaché temporaire d'enseignement et de recherches, kurz: ATER) an den Universitäten. Das bietet zwar früh einen gesicherten Lebensunterhalt, hat aber den Nachteil, das aufgrund der oft hohen Arbeitsbelastung nur bedingt Zeit für die Dissertation bleibt (auch wenn das Promovieren neben dem Schuldienst möglich bleibt). Die Einführung von besonderen Arbeitsverträgen zwischen Universitäten und Doktoranden (contrats doctoraux) soll also prekären sozialen Zuständen bzw. ungünstigen Promotionsbedingungen abhelfen, die nicht zu Unrecht für lange Dissertationszeiten und hohe Abbruchquoten verantwortlich gemacht werden. Als Folge wird es inzwischen aber zunehmend schwer, von typischen Ausbildungswegen abzuweichen und z. B. im Rahmen eines Seniorenstudiums zu promovieren. Auch ist die Zahl der vom Hochschulministerium bereit gestellten contrats doctoraux begrenzt, was die Anzahl der Promotionen gerade in den Geistes- und 
Sozialwissenschaften tendenziell wieder sinken lässt. Schließlich bringt die ungleiche Verteilung dieser Arbeitsverträge auf die einzelnen Hochschulen die Gefahr mit sich, dass sich Bewerberinnen und Bewerber bei der Wahl ihrer Betreuer nicht mehr an wissenschaftlichen, sondern an materiellen Kriterien orientieren. Wer in den Genuss eines solches Vertrags kommt, hat jedoch den nicht geringen Vorteil einer sozialversicherungspflichtigen Beschäftigung, die - im Gegensatz zu Stipendien - auf die Rente angerechnet wird und Zugang $\mathrm{zu}$ Sozialleistungen wie der Arbeitslosenunterstützung eröffnet (dafür allerdings auch steuerpflichtig ist).

Zum Promovieren in Frankreich gehört zum anderen, dass man - gerade in Paris und anderen Großstädten - mit vergleichsweise ungünstigen Arbeitsbedingungen rechnen muss $^{23}$. Nicht nur sind Wohnungen klein und teuer, auch viele Hochschulen leiden am Zustand des Immobilienmarkts. Wer aus Deutschland eigene Arbeitsplätze und Computer für Promovierende gewohnt ist, wird sich in Frankreich deshalb umstellen müssen. Dort haben oftmals nicht einmal die Hochschullehrerinnen und -lehrer eigene Büros, sondern teilen sich zu zweit oder zu dritt einen Schreibtisch. Mehr als einen kollektiven Arbeitsraum können deshalb die meisten Hochschulinstitute ihren Doktoranden nicht zur Verfügung stellen - und mitunter fehlt sogar dieser. Dann bleibt nur der Gang in die häufig überfüllten Bibliotheken, vor denen sich gerade in Examenszeiten nicht selten lange Schlangen bilden, weil dann auch die Studierenden nach Platz und Ruhe für die Prüfungsvorbereitung suchen. Von diesen Zuständen kann man sich abschrecken lassen oder aber sie lebensweltlich ins Positive wenden und es den zahllosen Schriftstellern, Philosophen und Journalisten gleichtun, die das intellektuelle Tagwerk auf ihrem Stammplatz im Café absolvieren. Bücherkoller und die Gefahr von Vereinsamung drohen so jedenfalls nicht.

Deutlich wird an diesen Schilderungen schon, dass die neuen, strukturierten Ausbildungsformen keinen grundsätzlichen Wandel in den Promotionsbedingungen herbeigeführt haben. Die Einbindung in den kollektiven Forschungsprozess erfolgt in Frankreich nach wie vor zuerst auf der Ebene der Forschungsinstitute, in die Doktorandinnen und Doktoranden immer stärker integriert werden. Auf der Ebene der konkreten Arbeitsbedingungen bleiben sie hingegen weitgehend auf sich selbst gestellt (auch wenn es zahlreiche Hilfs- und Unterstützungsangebote gibt, etwa für die Organisation von Archiv- und Forschungsaufenthalten im Ausland ${ }^{24}$. Auf intellektuellem Niveau schließlich hat die Beziehung zwischen Doktorand und Betreuer - im Französischen kennt man keine paternalistischen Bezeichnungen wie im Deutschen, sondern spricht ganz neutral vom directeur bzw. der directrice de thèse ${ }^{25}$ - nichts von ihrer zentralen Bedeutung eingebüßt. Allerdings spielt diese Verbindung im Vergleich $\mathrm{zu}$ Deutschland weniger stark eine Rolle für die weitere Karriereplanung (relevant sind in dieser Hinsicht vor allem kollektive Netzwerke, wie sie etwa beim Besuch der classes préparatoires oder beim Absolvieren der agrégation entstehen), und auch auf den Verlauf der Promotion haben die Betreuerinnen und Betreuern hier einen geringeren oder zumindest anderen Einfluss. Dies spiegelt sich auch darin wider, dass den Betreuern seit einiger Zeit verstärkt kollektive Betreuungsinstanzen (»comité de thèse« oder »comité de suivi de thèse«) zur Seite treten, die Doktorvater oder Doktormutter zwar nicht ersetzen, den Betreuungsprozess aber um andere Hochschullehrer erweitern. Allerdings geschieht das nur punktuell. intellektuellen Rahmenbedingungen. So ist es in Frankreich weitgehend unüblich, dass 
Betreuer bestimmte Themen »vergeben«. Im Regelfall kommen die Vorschläge von den Studierenden selbst, die sich oft bereits im Rahmen der Masterarbeit (bei einem Forschungsmaster) auf die Dissertation vorbereitet haben. Stärker kollektiv verankert ist die Themenwahl bei Forschungsverbünden wie etwa den deutschen Exzellenzclustern vergleichbaren Laboratoires d'excellence (Labex). Die Bewerberinnen und Bewerber für die dort ausgeschriebenen Doktorandenstellen werden gewöhnlich von allen beteiligten Wissenschaftlern gemeinsam ausgewählt. In beiden Fällen ist die Bindung eines Doktoranden an seinen Betreuer nicht so fest, wie dies in Deutschland der Fall zu sein pflegt. Bemerkbar macht sich hier zum einen noch einmal das Verständnis von Promovierenden als jeunes chercheurs, die zwar noch Anleitung benötigen, gleichzeitig aber schon eigenständig am Forschungsprozess teilnehmen. Und zum anderen führen die unterschiedlichen Strukturen des Universitätsbetriebs selbst $\mathrm{zu}$ weniger starren Beziehungen. In Frankreich stehen Professoren keinen Lehrstühlen vor, die kleinen Unternehmen gleichen. Junge Wissenschaftlerinnen und Wissenschaftler werden deshalb auch nicht frühzeitig und eng an eine bestimmte Person oder Gruppe gebunden, der sie in der Wahrnehmung der anderen dann zugerechnet werden. Vielmehr bewegen sie sich relativ frei in den sie umgebenden Strukturen und sagen in Vorstellungsrunden deshalb weniger: ich promoviere bei Professor XY, sondern eher: ich promoviere am Institut Z.

Paradoxerweise ist in Frankreich der Druck auf Promovierende, Eigeninitiative zu entwickeln und sich in kollektiven Forschungsstrukturen früh selbst zu behaupten, jedoch nicht so hoch wie in Deutschland. Zwar werden Doktorandinnen und Doktoranden den internationalen Gepflogenheiten gemäß auch hier inzwischen angehalten, noch während der Dissertation erste Publikationen vorzulegen, kleinere Tagungen $\mathrm{zu}$ organisieren oder Drittmittel z. B. für die Finanzierung eines Auslandsaufenthalts einzuwerben. Da es im französischen System traditionell aber unüblich war, dass Doktoranden schon wie kleine Wissenschaftsunternehmer agieren, sind die gestellten Anforderungen in diesem Bereich vergleichsweise geringer. Dabei spielt eine Rolle, dass für die Rekrutierung an den Universitäten bis heute nicht - wie in Deutschland - allein die Fähigkeiten in Forschung und Wissenschaftsorganisation den Ausschlag geben, sondern auch Kriterien wie der Besuch von grandes écoles und der Erwerb der agrégation. Sicher sind die Dinge in diesem Bereich im Fluss (in der Politik wird seit Jahren die Abschaffung der agrégation diskutiert und im Wissenschaftsbereich setzt sich zunehmend als zusätzlicher Schritt zwischen Promotion und erster fester Stelle die Post-doc-Phase durch, was tendenziell die Forschungsorientierung verstärkt), in der konkreten Einstellungspraxis jedoch zeigen die traditionellen Muster nach wie vor große Beharrungskräfte. Das gilt auch für die Mobilität von Doktorandinnen und Doktoranden. Im Gegensatz zu Deutschland, wo mit dem "Hausberufungsverbot« das Prinzip des Ortswechsels fest im System verankert ist, ist es in Frankreich kein Makel, vom Abitur bis zur Rente an einem Ort zu bleiben. Das gilt für die Provinz, aber auch für Paris, wo sich die französische Wissenschaft nach wie vor konzentriert. Von hier weg gehen die meisten nach der Promotion nur, weil die Stellen in der Hauptstadt die begehrtesten sind. Der Abschied ist in den meisten Fällen jedoch nur temporär - und die Hoffnung, bald zurückkehren zu können, zeigt nichts besser als der Umstand, dass auch, wer in Reims, Saint-Etienne oder Rennes eine Stelle bekommen hat, in der Regel einfach in Paris wohnen bleibt und pendelt.

Diese französischen "Eigenheiten « setzen sich bis in die Methoden und Inhalte der Dissertationen fort. Zum einen gilt in Frankreich nicht die in Deutschland eherne Regel, 
dass zwischen Promotion und Habilitation die Forschungsgegenstände und die behandelten Zeiträume wechseln müssen ${ }^{26}$. Bis vor kurzem war es hier durchaus üblich, dass ein mit der Dissertation begonnenes Thema anschließend ein ganzes Forscherleben bestimmte. Auch in dieser Hinsicht hat sich in den letzten Jahren einiges geändert heute wird etwa bei Bewerbungen auf Post-doc-Stellen zumindest eine neue Fragestellung oder eine substantielle Erweiterung des Terrains erwartet. Einen grundsätzlichen Bruch in der Wahl ihrer Forschungsobjekte vollziehen aber nach wie vor die wenigsten. Das hat wieder mit den Rekrutierungsgewohnheiten an den Universitäten $\mathrm{zu}$ tun: Während in Deutschland vor allem auf der Basis von künftigen Forschungsertragserwartungen eingestellt wird, verlässt man sich in Frankreich eher auf das bereits Geleistete. Wer sich hier mit einem völlig neuen Projekt vorstellte, würde verwunderte Blicke ernten und Fragen provozieren, wie man denn sicher sein könne, das Versprochene auch einhalten zu können. Dabei spielt auch eine Rolle, dass - wenn man von einigen stark forschungsorientierten Institutionen wie der EHESS absieht Forschungsfähigkeiten nicht die einzigen und wichtigsten Qualifikationen sind, die von Bewerberinnen und Bewerbern erwartet werden. Das hat den durchaus positiven Nebeneffekt, dass auf Kompetenzen in der Lehre weitaus stärker geachtet wird als in Deutschland - die bereits angesprochenen Tätigkeiten als ATER oder im Sekundarschulbereich während der Dissertation sind deshalb Zusatzqualifikationen, die sich bei der Bewerbung als sehr nützlich erweisen können.

In den Dissertationen selbst spiegeln sich diese Unterschiede z. B. dadurch wider, dass die Fragestellungen stärker empirisch und weniger theoretisch bestimmt sind. Hier geht es nicht zuerst darum, ein argumentatives Feld zu beherrschen, konzeptuelle Fähigkeiten nachzuweisen und sich mit einer starken These von der existierenden Forschung abzusetzen, sondern stärker um eine möglichst weitgehende Durchdringung der Quellen, die Erschließung eines bislang nicht bearbeiteten Terrains oder die detaillierte Darstellung eines Falls. Die Tendenz zur Gründlichkeit und zur Vollständigkeit (die histoire totale hat ihre Wurzeln nicht zufällig in Frankreich!) führt dabei mitunter zu (für deutsche Verhältnisse) absurden Resultaten wie Manuskripten in drei Bänden von je 350 Seiten (Anhänge und Bibliographie nicht mitgerechnet). Obwohl heute allein schon die äußeren Bedingungen dazu drängen, Themen zu bearbeiten, die überschaubar sind und sich in einem lesbaren Umfang bewältigen lassen, sind hier immer noch die Nachwirkungen der 1986 abgeschafften Thèse d'État (einer Art Super-Habilitation, an der mancher mehrere Jahrzehnte saß) oder die Tradition der großen historischen Regionalstudien (Lucien Febvre zur Franche-Comté, Pierre Goubert zum Beauvais, Emmanuel Le Roy Ladurie zum Languedoc) zu spüren. Und auch die klassische Unterscheidung zwischen der monographie, die der möglichst erschöpfenden Darstellung eines bestimmten Gegenstands gewidmet ist, und dem wissenschaftlichen Essay, in dem eher übergreifende und theoretisch-methodologische Fragestellungen aufgegriffen werden, spielt nach wie vor eine Rolle.

Schließlich hat die unterschiedliche Strukturierung des wissenschaftlichen Felds auch Folgen für die internationale Zusammenarbeit. Der Logik des permanenten Wechsels folgend, bleiben deutsche Promovenden, die z. B. zu Frankreich gearbeitet haben, diesem Land bei den folgenden Karriereschritten selten treu. In Frankreich hingegen hat das Interesse für ein bestimmtes Land oder eine bestimmte historische Region oft ein ganzes Leben lang Bestand. Spezialisten für deutsche, italienische oder spanische Geschichte forschen heute nicht mehr unbedingt über Jahrzehnte hinweg zum gleichen Thema, sich 
plötzlich für amerikanische oder chinesische Geschichte zu interessieren, käme ihnen jedoch nicht in den Sinn. Das hat einerseits zur Folge, dass historische Regionalstudien in der Tradition der aires culturelles eine größere Konstanz aufweisen als in Deutschland; über die Generationen hinweg haben sich in diesem Bereich vielfach beständige Forschergruppen oder sogar eigene Institute gebildet ${ }^{27}$. Andererseits bedeutet die Tendenz zur Stetigkeit (die sich ebenfalls auf die größte Gruppe französischer Historikerinnen und Historiker erstreckt, derjenigen nämlich, die zur französischen Geschichte arbeiten) aber auch eine größere intellektuelle Trägheit, die sich etwa in den aktuellen Defiziten der französischen Geschichtswissenschaft im Bereich der Globalgeschichte bemerkbar machen - selbst wenn manche diesen Umstand nicht ganz zu Unrecht als eine begrüßenswerte Resistenz gegen allzu schnell wechselnde Forschungsmoden verkaufen.

\section{Die cotutelle de thèse und ihre Herausforderungen}

Diese wenigen Elemente machen sicher schon deutlich, dass Dissertationen in Doppelbetreuung (thèses en cotutelle) eine Reihe von Kompatibilitätsproblemen aufwerfen. Diese betreffen weniger administrative oder technische Punkte - diese sind angesichts der großen Zahl von Verfahren, die in den letzten Jahren bereits durchgeführt wurden, weitgehend gelöst -, sondern berühren eher intellektuelle Probleme, akademische Traditionen oder Fragen der Berufswahl ${ }^{28}$. Wer ein solches Doppelbetreuungsverfahren beabsichtigt, sollte sich möglichst frühzeitig über die Voraussetzungen und administrativen Bedingungen informieren. In den meisten Hochschulen existieren heute einschlägige Vereinbarungen, auf die leicht zurückgegriffen werden kann. Beachtet werden muss, dass eine cotutelle in der Regel nur im ersten Jahr der Dissertation auf den Weg gebracht werden kann (was einleuchtet, wenn man den Sinn des Verfahrens ernst nimmt). Gleiches gilt für die einschlägigen Unterstützungsangebote, etwa die cotutelleStipendien der Deutsch-Französischen Hochschule. ${ }^{29}$

Sind diese administrativen Hürden überwunden, müssen einige formelle Fragen geklärt werden. Dazu gehört erstens die Länge der Dissertationsschrift, die - wie bereits angedeutet - in Deutschland und in Frankreich erheblich differieren kann. Zweitens muss man sich bewusst sein, dass die Formen der Verteidigung diesseits und jenseits des Rheins nicht die gleichen sind. Eine deutsche Verteidigung dauert etwa eine anderthalb Stunden und gleicht meist einer echten wissenschaftlichen Debatte mit Fragen, Antworten und wechselseitigen Diskussionen. Deshalb fließt sie in die Endbenotung der Dissertation ein. In Frankreich ist eine Verteidigung hingegen sehr stark ritualisiert, kann sich über Stunden hinziehen und besteht vor allem aus monologischen Vorträgen der Kommissionsmitglieder, auf die zu antworten der Kandidat oder die Kandidatin nur kurz eine Chance hat. Hier geht es weniger um ein wissenschaftliches Gespräch (auch wenn als äußerst wohltuend empfunden wird, wenn ein solches zustande kommt, wie es gerade bei cotutelle-Verfahren oft der Fall ist), auch nicht um die Festlegung einer Note, sondern vor allem um die Aushandlung des bereits angesprochenen rapport de soutenance, der für den weiteren Karriereweg von essentieller Bedeutung ist und als "unseriös" betrachtet wird, wenn er nicht mindestens zehn Seiten lang ist ... Das Prozedere stellt für alle Beteiligten (nicht nur für den Kandidaten oder die Kandidatin) also eine echte Geduldsprobe dar, danach aber ist es geschafft: Das französische System kennt weder Rigorosum noch Disputation oder sonstige zusätzliche Prüfungen. Hinzuweisen ist 
schließlich noch darauf, dass in cotutelle-Verfahren die Verteidigung zumindest in Teilen in beiden Sprachen stattfindet.

Hinsichtlich der Promotionsurkunde sind die rechtlichen Regelungen klar: Beide Universitäten verleihen gemeinsam den Doktorgrad und geben dem durch die Verleihung einer gemeinsamen Urkunde Ausdruck. Leider hat sich dieser Gebrauch noch nicht überall durchgesetzt. Auch hinsichtlich der Note lauern Fallstricke: Erhält ein Kandidat in Deutschland ein magna cum laude, ist das ein durchaus ehrenhaftes Prädikat, mit dem sich eine akademische Karriere fortsetzen lässt; in Frankreich hingegen gilt - wie oben angedeutet - alles unter très honorable avec félicitations als suspekt. Im Zweifelsfall sollten also beide Noten auf der Urkunde vermerkt werden.

Noch größeres Fingerspitzengefühl verlangen schließlich die informellen Unterschiede zwischen beiden Systemen, bei denen Promovierte häufig auf die eigenen diplomatischen Fähigkeiten zurückgeworfen sind, denn Regelwerke existieren in diesem Bereich natürlich nicht. Nicht selten sieht sich so ein Doktorand oder eine Doktorandin mit widersprüchlichen - inhaltlichen, theoretischen oder methodischen - Anforderungen von Seiten der beiden Betreuerinnen oder Betreuer konfrontiert. In Deutschland wird man etwa auf die rasche Erarbeitung einer Fragestellung drängen, während in Frankreich zunächst der Gang ins Archiv und der detaillierte Blick in die Quellen für dringend notwendig erachtet wird. Anschließend wird ein deutscher Doktorvater vielleicht auf rasche erste Ergebnisse hoffen, die in seinem Doktorandenkolloquium oder einer ersten Publikation vorgestellt werden können. Eine französische directrice de thèse hingegen wird womöglich den Wunsch nach einem detaillierten plan äußern, dessen (nicht nur rhetorische) Logik sich Deutschen nicht immer unmittelbar erschließen wird, liegen die subtilen Herausforderungen hier doch nicht nur in der Gliederung in drei (oder mehr) Teile, sondern auch in der Notwendigkeit, bereits frühzeitig ausgeklügelte Vorstellungen von den "transitions« - also den Übergängen von einem Kapitel zum nächsten vorzulegen.

Geschick und Vermittlungskünste sind vor allem dann gefragt, wenn die beiden Betreuer sich und das jeweils andere System nicht oder schlecht kennen und deshalb Schwierigkeiten ihren Promovierenden und nicht den Unterschieden in den beiden Wissenschaftskulturen zuschreiben. Deshalb ist anzuraten, eher zwei Betreuer zu wählen, für die eine cotutelle keine völlig neue Erfahrung ist (häufig besteht der Kontakt zunächst zu einem der beiden, der dann eventuell auf Kolleginnen und Kollegen verweisen kann, mit denen er bereits kooperiert). Vor allem aber sollte man sich von solchen Erfahrungen nicht entmutigen lassen - denn sie sind es, die die immer wieder beschworenen »interkulturellen Kompetenzen" entstehen lassen, die einen Großteil des Mehrwerts einer Doppelbetreuung ausmachen. Sie sollten deshalb in das eigene Nachdenken integriert und zum Ausgangspunkt einer produktiven Spannung genommen werden, die dabei behilflich ist, das Beste aus den beiden wissenschaftlichen Systemen und intellektuellen Welten für die eigene Arbeit fruchtbar zu machen.

31 Auch im Stadium des Schreibens werden die Anforderungen nicht unbedingt die gleichen sein. In Deutschland wird gewöhnlich ein »akademischer« Stil gepflegt, der Leserinnen und Lesern außerhalb der Wissenschaft nur bedingt verständlich ist. Wichtige Kriterien sind dabei Genauigkeit im Ausdruck und Präzision bei der Verwendung von Begriffen (weshalb es z. B. keine Schande ist, das gleiche Wort viermal hintereinander $\mathrm{zu}$ verwenden, wenn es das genau passende Wort ist), während jede literarische Ambition beim Schreiben rasch als verdächtig und »unwissenschaftlich" eingestuft wird. In 
Frankreich hingegen wird das Fehlen einer gewissen rhetorischen Eleganz sofort bemerkt und als unangenehm empfunden. Wer sich also entschließt, im Rahmen einer Doppelbetreuung seine Dissertation auf Französisch $\mathrm{zu}$ verfassen, sollte über ausreichende Sprachkenntnisse verfügen (und darf sich beim Gelingen ganz sicher auf lobende Worte von Seiten der Gutachter freuen). Gleiches gilt für die Anmerkungen in den Fußnoten: Während in Deutschland Gewissenhaftigkeit ganz groß geschrieben wird, darf es in Frankreich etwas freizügiger zugehen (von der Pflicht zur Beachtung der Regeln exakten Zitierens sind Promovierende natürlich aber auch hier nicht entbunden). Beim Wechseln zwischen den Sprachen - wie es allen Doktorandinnen und Doktoranden in Doppelbetreuung spätestens beim Verfassen der Zusammenfassung bevorsteht - gilt es also immer mit $\mathrm{zu}$ bedenken, dass dabei auch die akademischen Kulturen gewechselt werden ${ }^{30}$.

Hingewiesen werden soll schließlich noch auf die »internationalen « Dissertationen, wie sie etwa am Europäischen Hochschulinstitut in Florenz angefertigt werden können ${ }^{31}$. Auch hier findet die Betreuung durch Hochschullehrerinnen und -lehrer statt, die aus verschiedenen europäischen Ländern stammen. Die Arbeit selbst aber wird nicht an zwei verschiedenen Universitäten angefertigt, sondern innerhalb einer besonderen Institution. Am Ende der Dissertation hat der frisch Promovierte also kein Doppeldiplom und auch keinen italienischen Doktorabschluss in der Hand, sondern einen echten »internationalen« Doktorgrad. Auf dem (akademischen) Arbeitsmarkt in Frankreich hat dieser leider jedoch mit einigen Schwierigkeiten zu kämpfen, weil er beispielsweise mit keinem rapport de soutenance verbunden ist, wie ihn viele Universitäten nach wie vor für unerlässlich bei der Einstellung junger Kolleginnen und Kollegen erachten.

Zum Abschluss möchten wir Sie ermuntern: Trotz aller weiter bestehenden Unterschiede und Eigenheiten haben sich die akademischen Systeme in Deutschland und Frankreich nach zwei Jahrzehnten Harmonisierung im europäischen Hochschulraum erheblich angenähert. Die Möglichkeiten für Mobilität, Austausch und das Pendeln zwischen beiden Ländern haben heute eine Qualität erreicht, wie sie bislang in der europäischen Geschichte bestenfalls im Spätmittelalter existiert hat, als es (für eine kleine Geisteselite) gang und gäbe war, ein intellektuelles Dasein im Wechsel zwischen den großen Universitäten des Kontinents zu verbringen. Hunderte von deutschen Doktorandinnen und Doktoranden sind bereits den Weg eines Forschungsaufenthalts oder einer Promotion in Frankreich gegangen und haben die Chancen auf menschliche und kulturelle Bereicherung, intellektuelle Horizonterweiterung und ein Mehr an Reflexivität wahrgenommen, die eine solche Erfahrung mit sich bringt. Nicht wenigen von ihnen ist anschließend auch der Berufseinstieg als Hochschullehrerin oder Forscher in Frankreich gelungen - im Vergleich zu Deutschland, wo zumindest im Fach Geschichte ausländische Professorinnen und Professoren eine absolute Rarität darstellen, erweist sich das französische System in dieser Hinsicht trotz einiger Eigenheiten wie der agrégation als deutlich offener. Folgen Sie ihrem Beispiel mit Zuversicht: Denn wenn Wissenschaft heißt, unentwegt die eigenen Gewissheiten infrage zu stellen, dann lässt sich kein besseres Abenteuer vorstellen als ein Streifzug zwischen zwei akademischen Systemen mit ihren unterschiedlichen Formen und Praktiken des wissenschaftlichen Arbeitens, ihren verschiedenen disziplinären Traditionen und den vielfältigen formellen und informellen Regeln, mit denen Wissen und Kompetenzen hier jeweils in Diplome und Titel gegossen werden. 


\section{NOTES}

1. Einen guten Überblick über die Annales-Schule geben Lutz Raphael, Die Erben von Bloch und Febvre. Annales-Historiographie und nouvelle histoire in Frankreich 1945-1980, Stuttgart 1994 und Matthias Middell, Steffen Sammler (Hg.), Alles Gewordene hat Geschichte. Die Schule der Annales in ihren Texten 1929-1992, Leipzig 1994. Zur aktuellen Historiographie in Frankreich siehe beispielsweise Christian Delacroix, François Dosse, Patrick Garcia, Nicolas Offenstadt (Hg.), Historiographies. Concepts et débats. 2 Bde., Paris 2010 und Christophe Granger (Hg.), À quoi pensent les historiens. Faire de l'histoire au XXle siècle, Paris 2013.

2. Achtung vor weiteren »faux amis«: Der französische Begriff dissertation oder kurz »dissert steht nicht für die Doktorarbeit, sondern für eine schriftliche Abschlussarbeit oder Klausur; die Dissertation wird in Frankreich als thèse bezeichnet, während dem deutschen Wort »These« Bezeichnungen wie hypothèse oder argument principal entsprechen.

3. Vgl. dazu auch den Beitrag von Jean-Louis Georget "Karrieremöglichkeiten in der Wissenschaft und der Arbeitsmarkt für Historikerinnen und Historiker in Frankreich « in diesem Wegweiser ( http://ifha.revues.org/7940). Alle Links wurden zuletzt am 12.7.2014 überprüft.

4. Repères et références statistiques sur les enseignements, la formation et la recherche. Édition 2013, einsehbar unter: http://www.education.gouv.fr/statistiques/rers.

5. Vgl. Anna Karla, Nachwuchswissenschaft, jeune recherche et les »Mots de l'histoire«, in: Revue de l'Institut français d'histoire en Allemagne 4 (2012), S. 204-213 (http://ifha.revues.org/463).

6. Vgl. den Beitrag von Claire Gantet »Zwischen französischen Besonderheiten und internationaler Öffnung: Unterricht und Lehrerausbildung in Frankreich« in diesem Wegweiser ( http://ifha.revues.org/7857).

7. Vgl. Pierre Judet de la Combe, Crise de système dans l'enseignement supérieur français?, in: Paul Servais (Hg.), L'évaluation de la recherche en sciences humaines et sociales. Regards de chercheurs, Louvain-la-Neuve 2011, S. 55-82.

8. Commission permanente du Conseil national des universités (CP-CNU http://www.cpcnu.fr/; für die Geschichtswissenschaft zuständig sind die Sektionen 21: http://www.cpcnu.fr/web/ section-21/presentation und 22: http://www.cpcnu.fr/web/section-22/presentation Vgl. auch den Beitrag von Jean-Louis Georget "Karrieremöglichkeiten in der Wissenschaft und der Arbeitsmarkt für Historikerinnen und Historiker in Frankreich« in diesem Wegweiser (http:// ifha.revues.org/7940).

9. Arrêté du 7 août 2006 relatif à la formation doctorale, http://www.legifrance.gouv.fr/ affichTexte.do?cidTexte=JORFTEXT000000267752.

10. Das französische System kennt neben den Universitäten weitere Hochschuleinrichtungen, die sog. "grands établissements", die nicht "Universitäten" heißen, aber ebenfalls die Promotion verleihen. Für Historiker und Historikerinnen ist dieser Umstand besonders relevant, denn diese Institutionen bestehen vor allem in den Geistes- und Sozialwissenschaften. Dazu gehören die bereits erwähnten Écoles normales supérieures, aber auch einige prestigereiche Pariser Einrichtungen wie die angesprochene EHESS mit ihren Ablegern in Lyon, Marseille und Toulouse sowie ihre Schwesterinstitution, die École pratique des hautes études (EPHE). Darüber hinaus verleiht auch die École nationale des chartes, an der vor allem Archivare, Konservatoren und Bibliothekare ausgebildet werden, ein eigenständiges Doktordiplom. Diese "thèse de l'École des chartes«, die besonders für Mediävisten nach wie vor eine große Rolle spielt, wird in einigen Fällen als äquivalent zum allgemeinen Doktordiplom angesehen. Keine Diplome (also auch keine Promotionen) verleiht hingegen das Collège de France, ebenfalls ein »grand établissement«. 
11. CDFA »Construire les différences« - DFDK »Unterschiede denken«, http://www.differencesdifferenzen.eu, Forschungszentrum Historische Geisteswissenschaften - Collège doctoral francoallemand, http://www.fzhg.org/front_content.php?idcat=46. Für eine Gesamtliste der DeutschFranzösischen Doktorandenkollegs siehe DFH-UFA: Dt.-frz. Doktorandenkollegs (DFDK), http:// www.dfh-ufa.org/de/forschung/dt-frz-doktorandenkollegs-dfdk/.

12. Weitere Informationen unter http://www.ciera.fr (speziell zum Programme d'encadrement doctoral: http://www.ciera.fr/ciera/spip.php?rubrique79).

13. Deutsches Historisches Institut Paris, http://www.dhi-paris.fr/de/home/foerderungfellowships.html.

14. Institut français d'histoire en Allemagne, http://www.ifha.fr; Saisir l'Europe - Un réseau franco-allemand en sciences humaines et sociales, http://www.saisirleurope.eu.

15. Berufliche Eingliederung von Doktoranden: ABG-DFH: http://www.dfh-ufa.org/de/ forschung/abg/.

16. Les aspects concrets de la thèse, http://act.hypotheses.org/.

17. GIRAF-IFFD - Interdisziplinäre Forschungsgemeinschaft Frankreich-Deutschland, http:// www.giraf-iffd.eu/gs/.

18. Am längsten auf dem Markt - seit 1985 - ist das Buch von Michel Beaud, L'art de la thèse. Aktuelle Ausgabe (zus. mit Magali Gravier und Alain de Toledo): Comment préparer et rédiger un mémoire de master, une thèse de doctorat ou tout autre travail universitaire à l'ère du Net, Paris 2006.

19. ABES, http://www.abes.fr/Theses/Mission-nationale. Die ABES bietet eine Reihe von weiteren Serviceleistungen an, u. a. das Portal http://www.theses.fr/, eine spezielle Suchmaschine für alle aktuell in Arbeit befindlichen und abgeschlossenen Dissertationen in Frankreich.

20. Vgl. als konkretes Beispiel die Charte de thèse der Universität Paris 1 - Panthéon-Sorbonne: http://www.univ-paris1.fr/recherche/doctorat/etudes-doctorales/sinscrire-en-these/la-chartedes-theses.

21. Coût d'une inscription dans l'enseignement supérieur, http://vosdroits.service-public.fr/ particuliers/F2865.xhtml.

22. Atelier national de reproduction des thèses, http://www.diffusiontheses.fr/.

23. Vgl. dazu auch den Beitrag von Niels F. May »Einen Forschungsaufenthalt in Frankreich vorbereiten« in diesem Wegweiser (http://ifha.revues.org/7914).

24. Vgl. dazu auch den Beitrag von Delia Küsgen und Christiane Schmeken »Die Finanzierung eines Studien-, Lehr- und Forschungsaufenthaltes in Frankreich« in diesem Wegweiser (http:// ifha.revues.org/7919).

25. Promotionen betreuen darf in Frankreich nur, wer habilitiert ist (wobei der exakte Titel des Diploms, nämlich »habilitation à diriger des recherches« genau darauf hinweist). Darunter zählen also z. B. auch habilitierte Maître de conférences. Auch tragen nicht alle französischen Forscherinnen und Hochschullehrer mit Äquivalenz zum Professorenrang auch den Professorentitel. Im CNRS spricht man vielmehr von directeurs bzw. directrices de recherches sowie an der EHESS und der EPHE von directeurs bzw. directrices d'études.

26. Dabei variieren die in Deutschland und Frankreich jeweils üblichen Einteilungen der Geschichte in Großepochen z. T. erheblich. Die für die meisten französischen Historikerinnen und Historiker maßgebliche Zäsur ist die Revolution von 1789, die die Scheide zwischen »histoire moderne und »histoire contemporaine« markiert. Die deutsche »Frühe Neuzeit» bzw. "Neuzeit» entspricht dem jedoch nur bedingt (u. a. weil der in der deutschen Historiografie zentrale Einschnitt der Reformation in Frankreich weitgehend ohne Relevanz ist). Auch definieren sich viele französische Historikerinnen und Historiker zuerst als Spezialisten eines bestimmten Jahrhunderts (»dix-septièmiste«, »dix-huitièmiste« usw.) und nicht einer Epoche. Die in Deutschland verbreitete »Zeitgeschichte« als Periode nach 1917 bzw. 1945 schließlich setzt sich in Frankreich als »histoire du temps présent« nur langsam durch. 
27. Für den Bereich der deutschen Geschichte etwa das Centre Georg Simmel - Recherches franco-allemandes an der EHESS (http://centregeorgsimmel.ehess.fr/) oder die Forschergruppe »Pays germaniques« an der ENS Paris (http://www.umr8547.ens.fr/).

28. Die rechtlichen Grundlagen des cotutelle-Verfahrens werden in Frankreich vom »Arrêté du 6 janvier 2005 relatif à la cotutelle internationale de thèse» gelegt (http:// www.legifrance.gouv.fr/affichTexte.do?cidTexte=JORFTEXT000000258089). Über den rechtlichen Rahmen und zahlreiche technische Aspekte gibt darüber hinaus eine 2007 vom deutschfranzösischen Verein GIRAF-IFFD (Interdisziplinäre Forschungsgemeinschaft FrankreichDeutschland) durchgeführte Untersuchung Auskunft, die auch zahlreiche Berichte von Promovierten über ihre Erfahrungen mit dieser besonderen Form des Promovierens beinhaltet: http://www.giraf-iffd.eu/gs/index.php?id=enquetes-umfragen.

29. Deutsch-Französische Hochschule, http://www.dfh-ufa.org/de/forschung/promotioncotutelle-de-these/.

30. Siehe dazu auch den Beitrag von Anne Baillot und Franziska Heimburger »Geschichte schreiben: Französisch als Wissenschaftssprache» in diesem Wegweiser (http:// ifha.revues.org/7931).

31. Doctoral Programme - European University Institut, http://www.eui.eu/ServicesAndAdmin/ AcademicService/DoctoralProgramme/Index.aspx.

\section{RÉSUMÉS}

In Frankreich hat eine Promotion - zumindest außerhalb des engeren akademischen Feldes nicht den gleichen Stellenwert wie in Deutschland. Der Beitrag versucht deshalb zunächst, die Stellung und den sozialen Status einer Doktorarbeit in der französischen Gesellschaft zu umreißen. Im Anschluss daran werden die Strukturen der Doktorandenausbildung vorgestellt, die sich hier vor allem dadurch auszeichnen, dass sie weitgehend national organisiert sind. Ein Blick auf die für deutsche Promovierende in Frankreich besonders interessanten Betreuungsangebote leitet über zu einer Vorstellung der konkreten Promotionsbedingungen, wobei sowohl auf materielle als auch auf intellektuelle Aspekte eingegangen wird. Abschließend wird das Promovieren in Doppelbetreuung (cotutelle) thematisiert und ganz besonders auf die informellen Unterschiede zwischen dem deutschen und französischen System eingegangen.

\section{INDEX}

Schlüsselwörter : Frankreich, Universität, Geschichtswissenschaft, Geschichte , Promovieren, Dissertation, Cotutelle, Doktorgrad, Doktorandenausbildung, Promotionsbedingungen

\section{AUTEURS}

\section{FALK BRETSCHNEIDER}

Dr. Falk Bretschneider ist Maître de conférences an der École des hautes études en sciences sociales (EHESS) und Leiter des Europa-Programms und der deutsch-französischen 
Forschungskooperation an der Fondation Maison des sciences de l'homme in Paris.

Mail: Falk.Bretschneider[at]ehess.fr

\section{CHRISTOPHE DUHAMELLE}

Prof. Dr. Christophe Duhamelle ist Directeur d'études an der École des hautes études en sciences sociales Paris (EHESS).

Mail: Christophe.Duhamelle[at]ehess.fr 